\title{
Factores de riesgo biomecánico lumbar por manejo manual de cargas en el reparto de productos cárnicos
}

\author{
Lumbar biomechanical risk factors due to manual \\ handling of loads in the distribution of meat products
}

Carlos Ibarra-Villanueva ${ }^{1}$ (1) 0000-0002-2178-6212

Pamela Astudillo-Cornejo' (1) 0000-0002-1457-2072

'Universidad de Atacama, Facultad de Ciencias de la Salud, Departamento de Kinesiología, Copiapó, Chile.

Fechas · Dates

Recibido: 2020.11.20 Aceptado: 2021.08.02

Publicado: 2021.10.15
Correspondencia · Corresponding Author

Carlos Ibarra-Villanueva

carlos.ibarra@uda.cl 


\section{Resumen}

Introducción: Identificar los factores de riesgo de trastornos musculoesqueléticos (TME) lumbares asociados al manejo manual (MMC) de productos cárnicos en las actividades de carga y descarga de camiones.

Método: Estudio observacional descriptivo transversal con enfoque mixto, basado en el análisis ergonómico de la actividad de trabajo. Participaron los 4 trabajadores de los puestos de cargador y cargador-conductor. Se realizaron análisis cinemáticos y dinamométricos para determinar las fuerzas de compresión y cizallamiento y el riesgo biomecánico musculoesquelético en la zona lumbar. Se realizaron entrevistas y observaciones para analizar tareas y determinar los tiempos involucrados en cada operación, tipo de exposición y los determinantes de la actividad de trabajo.

Resultados: El contexto de trabajo es sumamente variable durante la carga y descarga del camión, considerando la variabilidad en cada punto de entrega las características de los estacionamientos, las condiciones climáticas, la presencia de animales y otros obstáculos durante el transporte manual de la carga. El MMC produce fuerzas sobre los $4000 \mathrm{~N}$ de compresión discal en los niveles L4-L5 y L5-S1, las fuerzas iniciales de empuje y arrastre fueron de $51.2 \mathrm{~kg} \star$ f y $27.3 \mathrm{~kg} \star$ respectivamente, lo que evidencia el riesgo de TME. La actividad de trabajo está determinada por el tipo de jornada, la organización del reparto y la falta de medios mecánicos de apoyo para la carga y descarga de los camiones.

Conclusiones: Las tareas de MMC en esta industria son físicamente muy exigentes y mejorar estas condiciones de trabajo representa un desafío importante para la prevención.

Palabras clave: Evaluaciones Ergonómicas, Enfermedades musculoesqueléticas, Riesgo Biomecánico, Manejo Manual de Cargas, Industria Cárnica.

\section{Abstract}

Introduction: Identify risk factors for lumbar musculoskeletal disorders (MSD) associated with manual handling (MHL) of meat products in truck loading and unloading activities.

Method: Cross-sectional descriptive observational study with a mixed approach, based on ergonomic analysis of work activity. The 4 workers from the loader and loader-driver positions participated. Kinematic and dynamometric analyzes were performed to determine compression and shear forces and musculoskeletal risk in the lumbar area. Interviews and observations were conducted to analyze tasks and determine the times involved in each operation, type of exposure and the determinants of work activity.

Results: The work context is highly variable during the loading and unloading of the truck, considering the variability at each delivery point the characteristics of the parking lots, weather conditions, the presence of animals and other obstacles during manual transport of the load. The MHL produces forces over $4000 \mathrm{~N}$ of disc compression at levels L4-L5 and L5-S1, the initial push and pull forces were $51.2 \mathrm{~kg} * f$ and $27.3 \mathrm{~kg}{ }^{\star}$ f respectively, which shows the risk of MSD. The work activity is determined by the type of working day, the organization of the distribution and the lack of mechanical means to support the loading and unloading of trucks. 
Conclusions: The manual handling tasks in this industry are physically very demanding and improving these working conditions represents a significant challenge for prevention.

Keywords: Ergonomic Assessment; Musculoskeletal Diseases; Biomechanical Risk; Manual Handling of Loads, Meat Industry.

\section{Introducción}

Los trastornos musculoesqueléticos (TME) representan una carga de enfermedad y económica considerable en el mundo, siendo el dolor lumbar una de las principales causas de discapacidad en la mayoría de los países ${ }^{(1)}$. En Chile los TME son el grupo de enfermedades más prevalentes en la población laboral, el año 2019 representaron el $43 \%$ de las enfermedades profesionales ${ }^{(2)}$. Sin embargo, se desconoce cuántas corresponden a lesiones de zona lumbares y cervical( ${ }^{(1,3)}$.Además, el MMC es una de las principales causas de lesiones graves en las industrias que genera importantes costos de atención médica y discapacidad(1,3).

Los factores de riesgo de TME son clasificados según el tipo de actividad de trabajo que se realiza. El trabajo repetitivo tiene como principal factor de riesgo la alta frecuencia de movimientos de las extremidades superiores, a lo cual se agrega la postura, la fuerza y la falta de pausas, en ciclos de trabajos que son breves y repetidos durante la jornada laboral ${ }^{(4)}$. Por otra parte, trabajos donde los operadores deben pasar largos periodos en posturas de trabajo estáticas con posturas forzadas mantenidas es otro tipo de exposición a riesgo de $\mathrm{TME}^{(4)}$. En ese sentido la International Organization for Standardization (ISO) define normas específicas para el trabajo repetitivo en la ISO 11.228-3, para el MMC especifica el levantamiento/ descenso y transporte manual de cargas en la ISO 11.228-1, el empuje y arrastre de cargas en la ISO 11.228-2 y el trabajo postural estático en la ISO 11.226. Estas normas han sido adaptadas en Chile y forman parte de la regulación vigente en Seguridad y Salud del Trabajo (SST) siendo obligatoria su implementación por las empresas tanto en la identificación, como en la evaluación y gestión del riesgo de TME. Además, en los últimos años, Chile ha adoptado las recomendaciones de la ISO-TR 12.295- 2014(4) donde el énfasis es la identificación de condiciones aceptables "sin riesgos" o condiciones críticas "intolerables", estas últimas para ser intervenidas de manera inmediata.

El MMC de grandes animales, particularmente de cuartos de vacuno, expone a los trabajadores a riesgos de lesiones por accidentes de trabajo y $\mathrm{TME}^{(5-8)}$. Los riesgos conciernen al conjunto de la cadena de producción, incluidas las plantas faenadoras, las líneas de desposte, la cadena de distribución, el transporte e incluso en las carnicerías $^{(5,7)}$. En ese sentido, el trabajo de los operadores que realizan la carga y descarga de los camiones de reparto los enfrenta a dificultades y exigencias muy variables dependiendo del contexto(5). Este tipo de trabajo, predominantemente manual con importante esfuerzo físico, es característico de trabajadores de cuello azul (trabajadores manuales), con altas demandas físicas ocupacionales, tiempo prolongado de pie alternando trabajo estático y dinámico (combinados con sos- 
tener o manipular cargas), con flexión de la espalda y elevación reiterada de los brazos sobre el nivel de los hombros, entre otros factores de riesgo a la salud(9). Los altos niveles de demanda de trabajo físico en estos grupos ocupacionales generan mayor incidencia de riesgo TME, enfermedad cardiovascular ${ }^{(10,11)}$ y mortalidad ${ }^{(12-14)}$, capacidad de trabajo reducida ${ }^{(15,16)}$, ausentismo por enfermedad ${ }^{(17)} \mathrm{y}$ abandono prematuro del mercado laboral(18,19).

En Chile, no hay antecedentes de estudios sobre los riesgos a los cuales se exponen los trabajadores que manipulan grandes animales ya que se han centrado más en los rubros de minería, otros tipos de industria y servicios ${ }^{(20)}$. El presente estudio busca identificar los factores de riesgo de TME lumbares asociados al MMC, en las actividades de carga y descarga de productos cárnicos para el despacho a carnicerías.

\section{Métodos}

Diseño: Estudio de caso de tipo observacional descriptivo de corte transversal, con aproximación mixta. El estudio ergonómico se centra en el análisis de la actividad de trabajo ${ }^{(21,22)}$ de los operadores de carga y descarga en el reparto de productos cárnicos a carnicerías en una empresa faenadora de grandes animales en el sur de Chile el año 2019.

Participantes: 4 empleados hombres de 30 a 46 años (38 \pm 7.3$), 3.9( \pm 1.3)$ años trabajando en la empresa, peso $84.8 \mathrm{~kg}( \pm 11.6)$, talla $1.70 \mathrm{~m}( \pm 1.5)$ e índice de masa corporal (IMC) de $29.3( \pm 3.7)$ participaron voluntariamente previo consentimiento oral y escrito, respetando los principios de la Declaración de Helsinki, respecto a la privacidad y confidencialidad durante todo el proceso.

Observaciones y verbalizaciones: Se realizaron observaciones durante 3 jornadas laborales completas, filmación con dos cámaras marca Cannon VIXIA® modelo HFR800, obteniendo siempre dos planos de cada registro. Los que fueron analizados con el Software Captiv ${ }^{\circledR}$ v. L 2100, para determinar los tiempos de cada actividad y tipo de exposición a riesgo por cada operación. La velocidad de análisis de cada video fue a $0.125 X$. Se realizaron verbalizaciones directas con los trabajadores durante su actividad para conocer su apreciación respecto a la carga de trabajo y las posibilidades de mejoras.

Carga de trabajo biomecánico: Se utilizó software Kinovea® versión 0.8.15 para determinar el desplazamiento angular de segmentos corporales durante las operaciones de MMC y luego el Software 3D SSPP ${ }^{\text {TM }}$ V7.0.5 University of Michigan ${ }^{\circledR}$ para valorar la fuerza de compresión y cizallamiento en la zona lumbar baja(23,24), tomando como referencia los valores límites establecidos para compresión por el Instituto Nacional para la Seguridad y Salud Ocupacional (NIOSH) de Estados Unidos ${ }^{(25)}$, así como otros de consenso para las fuerzas de cizallamiento(26). El análisis cinemático se realizó a partir de las secuencias de video obtenidas para cada trabajador del estudio y sus resultados se expresan en valores promedios. 
Para el empuje y arrastre de cargas se midió la fuerza inicial con un dinamómetro SHIMPO®, modelo FGV-XY 500, luego se comparó el valor obtenido con el de las Tablas de Liberty Mutual ${ }^{(27)}$, considerando la condición desplazamiento de $2.1 \mathrm{~m}$, para 8 h de trabajo, una altura de aplicación de la fuerza de $144 \mathrm{~cm}$ para hombres y con criterio de protección del $90 \%$ de los trabajadores, teniendo presente que este método cuantitativo para empuje o arrastre de cargas está incluido en la norma chilena( ${ }^{(28)}$. Además, se cuantificó el peso de las cargas con una balanza industrial modelo 14191-489F. Se demandó también la percepción del esfuerzo por medio de la Escala de Borg modificada de 10 puntos.

Los datos obtenidos se ingresaron a planillas Excel® v.2019 y luego se utilizó para el análisis estadístico descriptivo el Software SigmaPlotß versión 12.

\section{Resultados}

Descripción de la Actividad de Trabajo: En ambas tareas de carga y descarga del camión, tanto el cargador como el conductor realizan MMC al transportar los Cuartos Anterior Vacuno (73 kg), Cuarto posterior (81 kg), Cabeza $(16 \mathrm{~kg})$, Cordero (16 kg), Caja con vísceras (60 kg), Gancho (3 kg), más herramientas ganchos y cajas vacías.

Durante la carga del camión, ambos trabajadores del turno (conductor y cargador) realizan las mismas tareas. El trabajo se desarrolla en un espacio circunscrito a las líneas de despacho, donde existe un desnivel de $1.5 \mathrm{~m}$ de altura entre la plataforma que está a la altura del camión y la zona de carga con los rieles desde el frigorífico. Ambos trabajadores, deben manipular las piezas de carne (Figura 1A), empujándolas por los rieles y/o transportándolas manualmente sobre un hombro (Figura 1A -1), además de cortar cada mitad del vacuno colgado en el riel, a la altura de la novena o décima costilla con un serrucho y un cuchillo, mientras lo sostiene con un brazo y hombro (Figura 1A -2 y 3). Luego debe empujar el cuarto anterior del vacuno para levantarlo, ponerlo sobre sus hombros y cortar, y luego caminar cargando los cuartos de vacuno que pesan entre 73 y $81 \mathrm{~kg}$, subiendo una pendiente ( $3 \mathrm{~m}$ de longitud y $18^{\circ}$ de inclinación) hacia el camión (Figura $1 \mathrm{~A}-4$ y 5), donde deposita el cuarto sobre un gancho en el riel dentro del camión. Esto en pisos húmedos por los fluidos y la limpieza con agua, para lo cual se dispone de un tapiz de goma en la rampa.

Los cuartos posteriores del vacuno que permanecen en los rieles se empujan para que queden cerca del camión, luego lo desenganchan sosteniéndolo y lo transportan sobre sus hombros hasta engancharlo al interior del camión.

En la segunda tarea que es la descarga del camión en el despacho a las carnicerías, ambos trabajadores deben manipular manualmente nuevamente las piezas cárnicas (Figura 1B), no obstante, los roles de ambos son diferentes. Respecto de los vacunos, el conductor empuja las piezas cárnicas en los rieles del camión para acercarlas a la puerta donde espera el cargador (Figura 1B-1). Para esto el conductor desengancha la pieza desde el riel y la sostiene manualmente con los 
ganchos para depositarla suavemente sobre el hombro del cargador (Figura 1B-1). Es un esfuerzo que ambos conductores califican como "muy duro" con 8 puntos en la escala de Borg e implica un esfuerzo muscular estático al sostener la carga por algunos segundos. Luego el cargador levanta y transporta manualmente el cuarto de vacuno desde el camión a la zona de descarga de cada carnicería en la ruta prevista (Figura 1B-2 y 3), en ese lugar desciende la carga de sus hombros y la engancha donde le señala el comprador, habitualmente en un frigorífico con rieles y ganchos (Figura 1B-4).El contexto es en extremo variable, considerando el estacionamiento del camión, las condiciones climáticas, la presencia de animales callejeros (perros) y obstáculos que se presentan durante la marcha a pie que realiza el trabajador (Figura 1B-3).

1A. Operaciones en actividad de carga de camión en la planta Faenadora
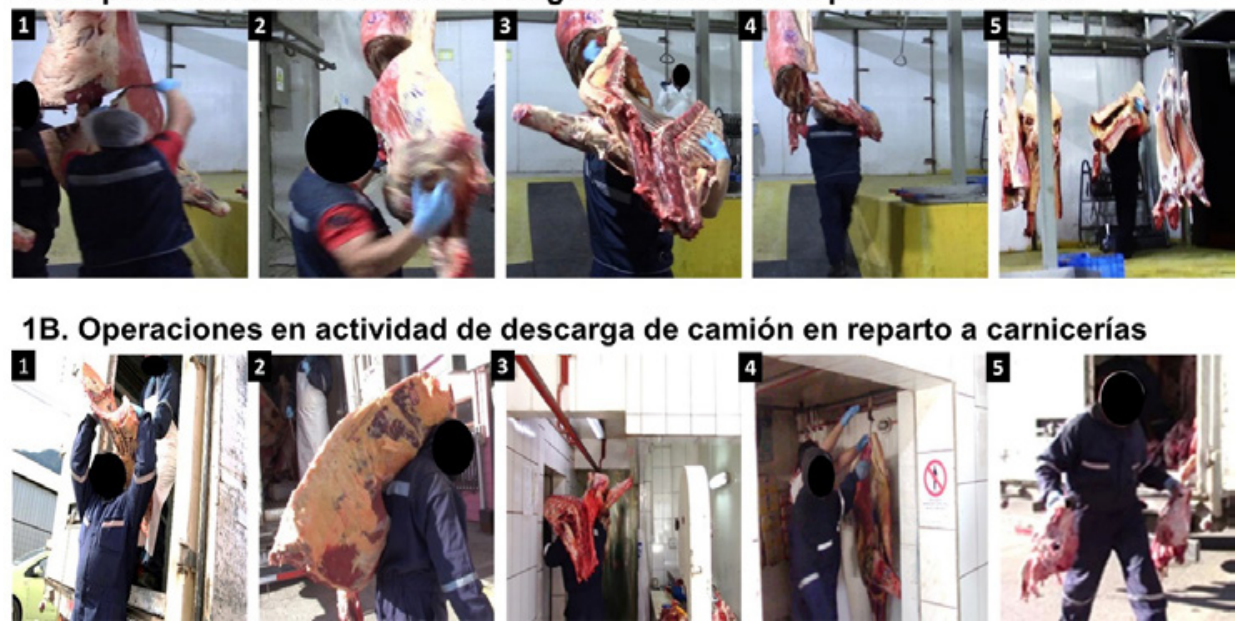

Figura 1: Operaciones de manejo manual en actividad de carga y descarga de cuartos de vacunos.

Por otra parte, las cajas con vísceras que pesan $60 \mathrm{~kg}$, son transportadas en equipo por ambos trabajadores, mientras que las cabezas las transportan ambos, pero de manera individual (Figura 1B-5).

A las actividades manuales antes descritas en la tarea de descarga, se agrega la actividad cognitiva asociada al control de la orden de despacho respecto de lo que se está entregando y en algunos casos la recepción de dinero en efectivo por pagos pendientes.

\section{Número y Tiempo promedio de las operaciones de MMC en Carga y Descarga:}

En la Tabla 1, se observa la duración unitaria de cada operación en el MMC, siendo levantar, transportar y descender, de mayor duración en la descarga del camión, comparativamente con la carga del camión en la planta, mientras que las operaciones de empuje y arrastre fueron siempre de mayor duración en la carga del camión. Levantar las cargas tuvo una duración de $1.3 \mathrm{~s}( \pm 0.1 \mathrm{~s})$ en la carga del camión y 1,9s ( $\pm 0.5 \mathrm{~s})$ en la descarga; transportar manualmente 7.1s (0.5s) en la carga y $11.2 \mathrm{~s}( \pm 2,1 \mathrm{~s})$ en la descarga; descender la carga $2.7 \mathrm{~s}( \pm 1.3 \mathrm{~s})$. Las operaciones de empujar y arrastrar fueron de mayor duración promedio en la carga 
del camión con $2.6 s( \pm 0.7 s)$ y $2.2 s( \pm 0.9 s)$ respectivamente. No se constató una diferencia estadísticamente significativa $(P=0,841)$ con el T-test.

Tabla 1: Número y tiempo promedio de las operaciones de manejo manual de cargas en la tarea de carga y descarga del camión

\begin{tabular}{lccccccc}
\hline & \multicolumn{7}{c}{ Tarea } \\
\cline { 2 - 6 } Operaciones & \multicolumn{5}{c}{ Carga Camión } & \multicolumn{3}{c}{ Descarga Camión } & \\
\cline { 2 - 6 } & $\mathbf{N}^{\circ}$ & $\begin{array}{c}\text { Promedio } \\
\text { Tiempo (s) }\end{array}$ & $\begin{array}{c}\text { Desviación } \\
\text { Estándar } \\
\text { Tiempo (s) }\end{array}$ & $\mathbf{N}^{\circ}$ & $\begin{array}{c}\text { Promedio } \\
\text { Tiempo (s) }\end{array}$ & $\begin{array}{c}\text { Desviación } \\
\text { Estándar } \\
\text { Tiempo (s) }\end{array}$ & \\
\hline Levantar & 60 & 1.3 & 0.0 & 102 & 1.9 & 0.5 & 0.841 \\
Transportar & 84 & 7.1 & 0.5 & 83 & 11 & 2.1 & 0.841 \\
Descender & 55 & 1.6 & 0.3 & 95 & 2.7 & 1.3 & 0.841 \\
Empujar & 112 & 2.6 & 0.7 & 2 & 1.4 & 0.0 & 0.730 \\
Arrastrar & 23 & 2.2 & 0.9 & 4 & 1.7 & 0.4 & 0.730 \\
\hline
\end{tabular}

También en la Tabla 1, se observa que en la descarga del camión el número de levantamientos $(n=102)$ y descensos $(n=95)$ es considerablemente mayor a los que se realizan en la carga del camión. Esto se debe a que en reiteradas ocasiones se comenten errores en la entrega de los productos donde la orden de despacho no coincide con lo que pidió el cliente, por lo cual el trabajador debe volver con la carga al camión. También se debe a que las cajas con vísceras y las cabezas de vacuno, cuando se carga el camión en la planta son arrastradas en contenedores, sin embargo, en el reparto, se sacan según el pedido del cliente de manera unitaria.

Por contraparte, se observa un número mayor de operaciones de empuje (112) y arrastre (23) en la carga del camión. Esto porque en la planta, los cuartos posteriores cuelgan de los rieles y los trabajadores los empujan hasta las cercanías del camión. Sin embargo, en terreno esta actividad no está presente. No se constató una diferencia estadísticamente significativa en el número de operaciones de carga y descarga con el T-test $(p=0,730)$.

Volumen de carga de una jornada de trabajo: El volumen obtenido a partir del peso estimado para cada pieza cárnica y objetos manipulados, multiplicado por la cantidad de veces que es transportado en la carga y descarga del camión. Esto se obtiene con la observación directa y se contrasta con las guías de despacho. La carga transportada representó un volumen total de $6844 \mathrm{~kg}$, que se dividen en $3422 \mathrm{~kg}$ en la fase de carga del camión y luego $3422 \mathrm{~kg}$ en la descarga en las carnicerías, representando desplazamientos mayores a los $20 \mathrm{~m}$ entre la distancia del camión y la zona de descarga de las carnicerías.

Análisis Cinemático y Momento de Fuerza Lumbar: Se realizó a partir de las secuencias de video obtenidas para cada trabajador del estudio en las tareas de levantamiento, descenso y transporte de cargas. Se utilizó el software Kinovea ${ }^{\circledR}$ para obtener los ángulos absolutos de movimiento de cada segmento (Figura 2A). Estos se ingresan junto a los datos antropométricos y las cargas manipuladas 
al Software 3D SSPP ${ }^{\text {TM}}$, con una imagen de referencia de la operación en análisis (Figura 2B). Con esto se determinan las fuerzas de compresión y cizallamiento en la zona lumbar baja, para cada tarea.

2A. Análisis en Software Kinovea para determinar ángulos absolutos

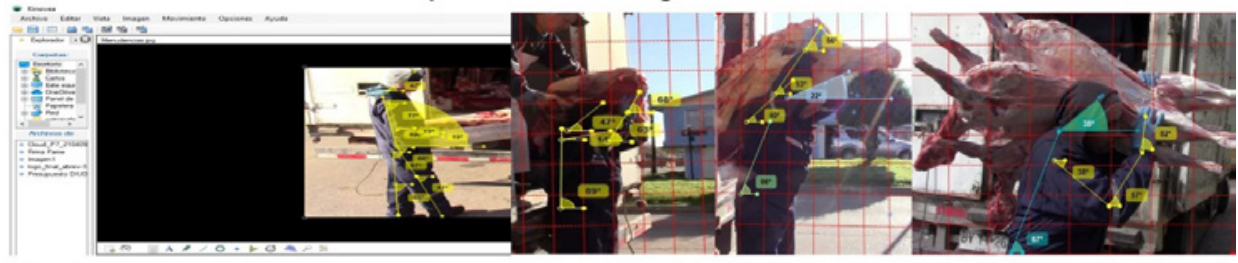

2B. Análisis en Software 3D SSPP para valorar la fuerza de compresión y cizallamiento

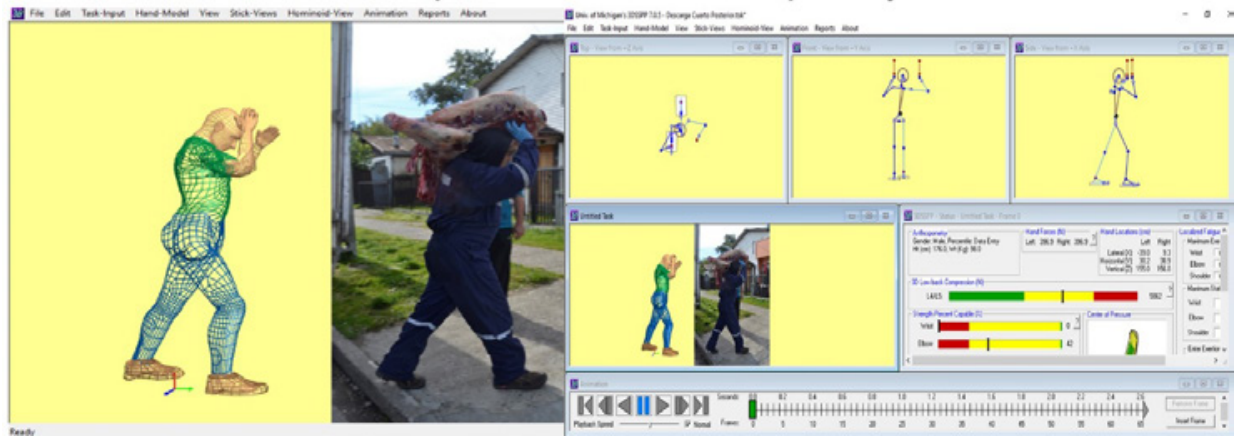

Figura 2: Análisis cinemático y momento de fuerza lumbar en las operaciones de manejo manual de cargas en actividad de carga y descarga de cuartos de vacunos desde el camión

En la Tabla 2 se puede constatar que en promedio las cargas superan los $3440 \mathrm{~N}$ que es el límite del $\mathrm{NIOSH}^{(25)}$, con un promedio de $4177 \mathrm{~N}( \pm 1130 \mathrm{~N})$ como fuerza de compresión en el nivel lumbar L4-L5 y 4007 N ( \pm 714 N) en L5-S1, lo que representa un riesgo significativo de daño de los discos intervertebrales de los trabajadores. En el caso de las fuerzas de cizallamiento, estas presentaron valores por debajo del límite de $1000 \mathrm{~N}$ recomendado(26).

Tabla 2: Fuerzas de compresión y cizallamiento lumbar en los niveles L4-L5 y L5-S1 para cada tarea de levantamiento

\begin{tabular}{lccccc}
\hline \multirow{2}{*}{ Tarea Levantamiento } & $\begin{array}{c}\text { Peso } \\
\text { Unitario } \\
(\mathbf{k g})\end{array}$ & \multicolumn{2}{c}{ Fuerza de Compresión $(\mathbf{N})$} & \multicolumn{2}{c}{$\begin{array}{c}\text { Fuerza de Cizallamiento } \\
\text { (N) }\end{array}$} \\
\cline { 3 - 6 } & 81 & $58448^{\mathrm{a}}$ & $467^{2} \mathrm{a}$ & 39 & 923 \\
\hline Cuarto Vacuno Posterior & 73 & $4143^{\mathrm{a}}$ & $4047^{\mathrm{a}}$ & 253 & 816 \\
Cuarto Vacuno Anterior & 16 & $4323^{\mathrm{a}}$ & $4557^{\mathrm{a}}$ & 387 & 598 \\
Coderos & 16 & 2687 & 2838 & 278 & 551 \\
Cabezas & 60 & $3886^{\mathrm{a}}$ & $3988^{\mathrm{a}}$ & 137 & 499 \\
Caja con Vísceras & 49.2 & $4177^{\mathrm{a}}$ & $4007^{\mathrm{a}}$ & 219 & 677 \\
Promedio & \pm 31 & \pm 1130 & \pm 714 & \pm 134 & \pm 183 \\
Desviación Estándar & & & & &
\end{tabular}

asupera el límite de compresión de $3400 \mathrm{~N}$ recomendado por NIOSH(25) 
Las operaciones que generan mayor compresión es el levantamiento del cuarto posterior de vacuno $(81 \mathrm{~kg})$ con 5844N para L4-L5 y 4612N para L5-S1; luego el levantamiento de 2 corderos simultáneamente $(30 \mathrm{~kg})$ con una compresión de $4557 \mathrm{~N}$ en L5-S1 y con 4323N en L4-L5 y el levantamiento del Cuarto Anterior de Vacuno (76 kg) con 4143N para L4-L5 y 4041N para L5-S1.

Empuje y arrastre de cargas: Los resultados de las fuerzas iniciales de empuje y arrastre medidas con dinamómetro, permitieron evidenciar que los trabajadores al empujar con ambas manos, sobre el nivel de la cabeza, la mitad de un vacuno colgado en el riel (160 kg), es de $51.2 \mathrm{~kg} \star \mathrm{f}$, superando los 31 kg*f que es el límite aceptable ${ }^{(27,28)}$. En el arrastre de la caja de vísceras $(60 \mathrm{~kg})$, la fuerza inicial es de $27.3 \mathrm{~kg}^{\star f}$ siendo el límite de $23 \mathrm{~kg}^{\star f}$ por lo cual en ambos casos se sobrepasa el máximo aceptable para una jornada de trabajo. Los resultados se expresan en $\mathrm{kg}^{\star} \mathrm{f}$ dado que en las tablas de Liberty Mutual en la norma chilena se expresan en esas unidades(28).

\section{Discusión}

En el estudio se evidenció una importante exposición a riesgo de los trabajadores, tanto conductores como cargadores, ya que ambos realizan constantemente MMC durante la jornada laboral. Las fuerzas de compresión lumbar y las exigencias en empuje y arrastre de cargas muestran la magnitud de la exigencia musculoesquelética. Los resultados de este estudio coinciden con la evidencia previa disponible en América del Norte y Europa ${ }^{(5-9)}$, en cuanto a la carga de trabajo físico ${ }^{(5-8)}$ y el elevado riesgo de $\operatorname{TME}^{(13,29)}$, sobre todo a nivel lumbar ${ }^{(21-26)}$ y en extremidades superiores ${ }^{(0,11)}$. Esto puede ser reducido con mecanización o automatización de rieles, cambio de canales de tubular a bi-riel, un brazo hidráulico de carga de camión, carros de transporte de $1 / 4$ de vacuno y corderos para descarga, o incluso el uso de exoesqueletos, entre otros ${ }^{(5,6,30,31)}$ y al centrar la intervención ergonómica en determinantes de la organización del trabajo tales como los ciclos de trabajo y descanso(3-6).

Entre las limitaciones del estudio se encuentran el peso de la carga que sobrepasa el límite máximo de $25 \mathrm{~kg}$ para hombres ${ }^{(28)}$, ya que muchos de los métodos para evaluar el riesgo referidos en la norma chilena ${ }^{(28)}$ no son aplicables a este tipo de condiciones más complejas, como la Ecuación de $\mathrm{NIOSH}^{(25)}$ por el peso que no puede exceder los $23 \mathrm{~kg}^{(25)}$ y el método MAC y V-MAC de la HSE ${ }^{(28)}$, por la manipulación que debe ser con ambas manos ${ }^{(28)}$, lo que deja fuera una gran cantidad de situaciones de trabajo cuyo riesgo debe ser demostrado y cuantificado de algún modo para hacerlo visible y gestionado. Por esta razón se recurrió al análisis de momento de fuerza para determinar las fuerzas de compresión y cizallamiento lumbar, método que es reconocido en el campo de la biomecánica ocupacional(21,24) y la ergonomía (21,22), aun cuando existen limitaciones como la exactitud del plano utilizado por la cámara durante una actividad dinámica o las bases antropométricas en el modelo biomecánico que se utiliza, en este caso el Software de la Universidad de Michigan ${ }^{(24)}$. Otra limitación fue la cantidad de unidades de análisis (4 trabajadores), aunque si bien representan al 100\% de trabajadores que realizan 
estas tareas en la empresa estudiada, no hace posible extrapolar la realidad de este centro de trabajo a otros, pero si expresa una realidad que puede ser cercana, sobre todo en empresas de menor tamaño, donde al igual que en este caso la polifuncionalidad de los operadores de descarga y los conductores es común. Por otra parte, el IMC promedio de los trabajadores de $29.3 \mathrm{~kg} / \mathrm{m}^{2}$, es una variable importante en las fuerzas de carga espinal, dado que se encuentra en el límite del sobrepeso. Los cambios en el peso corporal influyen en las cargas espinales, un mayor peso corporal produce cargas espinales más grandes y acumulación de grasa en la parte superior del tronco en comparación con más peso alrededor y debajo de la cintura pueden predisponer a las personas a un mayor riesgo de trastornos de la espalda ${ }^{(32)}$. Esta variable no fue parte de los análisis, dado que no se conoce cuales son las diferencias en la composición corporal de cada uno de los trabajadores (composición de masa grasa o masa magra), lo cual representa una limitante del estudio. Los individuos obesos con una circunferencia de cintura más grande (es decir, obesidad abdominal) experimentan mayores cargas, a aquellos solo tienen mayor peso por aumento de su masa muscular, por lo cual el papel de la forma del cuerpo (la forma de obesidad o somatotipos) ${ }^{(33)}$ debe ser considerada en futuros estudios. Esto no resta importancia a la magnitud de la exposición a factores de riesgo biomecánico en las condiciones de trabajo manual analizada.

Es necesario realizar investigaciones complementarias de mayor alcance en número de trabajadores y empresas en futuras investigaciones. Los hallazgos del presente trabajo contribuyen en aportar información sobre las condiciones de trabajo y el esfuerzo físico en el MMC de cuartos de bovinos, lo que permite alertar de su riesgo y de la falta de gestión preventiva en este sector de actividad.

Finalmente, considerar que la competencia de precios y la búsqueda continua de las ganancias de productividad que acompañan a este tipo de trabajos, tienen efectos directos sobre las condiciones de trabajo y la salud de los trabajadores ${ }^{(5,6)}$. El problema de los TME en los establecimientos de procesamiento de carne no es nuevo y las acciones de prevención de los riesgos deben ser prioritarias ${ }^{(5-8)}$, tal como hoy se relevan en esta industria las condiciones de inocuidad alimentaria, debieran relevarse también las condiciones de trabajo y particularmente las de MMC. Mejorar estas condiciones de trabajo y la regulación de estos aspectos en el momento de autorizar el funcionamiento de las carnicerías y las plantas faenadoras, representa un desafío de política pública sanitaria y para quienes se dedican a la prevención de riesgos, la ergonomía y a la producción.

\section{Financiación}

Este trabajo no cuenta con financiamiento externo a la Universidad de Atacama.

\section{Conflicto de intereses}

Los autores declaran no tener ningún tipo de relaciones económicas o de otra naturaleza que puedan haber influido en la realización del estudio y/o en la preparación de su trabajo para publicación. 


\section{Bibliografía}

1. Vos T, Allen C, Arora M, Barber RM, Brown A, Carter A, et al. Global, regional, and national incidence, prevalence, and years lived with disability for 310 diseases and injuries, 1990-2015: a systematic analysis for the Global Burden of Disease Study 2015. Lancet. 2016; 388: 1545-1602. doi:10.1016/S0140-6736(16)31678-6

2. Superintendencia de Seguridad Social de Chile [www.suseso.cl]. Santiago: Informe Estadísticas de Accidentabilidad 2019 [actualizado 27 jul 2021; citado 27 Jul 2021]. Disponible en: https://www.suseso.cl/607/w3-propertyvalue-10361.html

3. Stock SR, Nicolakakis N, Vézina N, Vézina M, Gilbert L, Turcot A, et al. Are work organization interventions effective in preventing or reducing work-related musculoskeletal disorders? A systematic review of the literature. Scand J Work Environ Health. 2018;44(2):113-133. doi:10.5271/sjweh.3696

4. International Organization for Standardization [www.iso.org]. ISO/TR 12295:2014 - Ergonomics - Application document for International Standards on manual handling (ISO 11228-1, ISO 11228-2 and ISO 11228-3) and evaluation of static working postures (ISO 11226). ISO. 2014. [actualizado 01 Abr 2014; citado 27 Jul 2021]. Disponible en: https://www.iso.org/standard/51309.html

5. Institute National de Recherche en Santé et Sécurité de France [www.inrs.fr]. Pour améliorer le transport et la livraison des carcasses de viande INRS France. 2016. [actualizado 01 May 2016; citado 27 Jul 2021]. Disponible en: http://www. inrs.fr/media.html?refINRS=ED\%206252

6. Toulouse G, Vézina N, Geoffrion L. Étude descriptive des déterminants des facteurs de risque de LATR aux postes d'éviscération abdominale de deux abattoirs de porcs. [online]. IRSST Québec. 1995. [actualizado 01 Sep 1995; citado 27 Jul 2021]. Disponible en: https://www.irsst.qc.ca/publications-et-outils/publication/i/470/n/etude-descriptive-des-determinants-des-facteurs-de-risque-de-latr-aux-postes-d-evisceration-abdominale-de-deux-abattoirs-de-porcs-r-108

7. Tirloni AS, dos Reis DC, Dias NF, Moro ARP. Ergonomic Risk Evaluation of the Manual Handling Task of Bovine Quarters in a Brazilian Slaughterhouse. En: Goonetilleke R, Karwowski W, editores. Advances in Physical Ergonomics and Human Factors. AHFE 2019. Washington: Springer; 2020. p. 57-69. doi:10.1007/978-3030-20142-5_6

8. Vergara LGL, Pansera TR. Ergonomics Analysis of the Activity of Boning Shoulder in a Pig Slaughter-house in the City of Ipiranga-SC. Work. 2012; 41: 703-709. doi:10.3233/WOR-2012-0229-703

9. Jørgensen MB, Gupta N, Korshøj M, Lagersted-Olsen J, Villumsen M, Mortensen OS, et al. The DPhacto cohort: An overview of technically measured physical activity at work and leisure in blue-collar sectors for practitioners and researchers. Applied Ergonomics. 2019; 77: 29-39. doi:10.1016/j.apergo.2019.01.003.

10. Krause N, Brand RJ, Kaplan GA, Kauhanen J, Malla S, Tuomainen T-P, Salonen JT. Occupational physical activity, energy expenditure and 11-year progression 
of carotid atherosclerosis. Scand J Work Environ Health. 2007; 33(6): 405-424. doi:10.5271/sjweh.1171

11. Wang A, Arah OA, Kauhanen J, Krause N. Effects of leisure-time and occupational physical activities on 20-year incidence of acute myocardial infarction: mediation and interaction. Scand J Work Environ Health. 2016; 42(5): 423-434. doi:10.5271/sjweh.3580

12. Holtermann A, Mortensen OS, Burr H, Søgaard K, Gyntelberg F, Suadicani P. The interplay between physical activity at work and during leisure time - risk of ischemic heart disease and all-cause mortality in middle-aged Caucasian men. Scand $J$ Work Environ Health. 2009; 35(6): 466-474. doi:10.5271/sjweh.1357

13. Punnett L. Musculoskeletal disorders and occupational exposures: how should we judge the evidence concerning the causal association?. Scand J Public Health. 2014; 42(13 Suppl): 49-58. doi:10.1177/1403494813517324

14. Holtermann A, Mortensen OS, Burr H, Søgaard K, Gyntelberg F, Suadicani P. Physical demands at work, physical fitness, and 30-year ischaemic heart disease and all-cause mortality in the Copenhagen Male Study. Scand J Work Environ Health 2010; 36(6): 466-472. doi:10.5271/sjweh.3120

15. Saidj M, Jørgensen T, Jacobsen RK, Linneberg A, Aadahl M. Differential cross-sectional associations of work- and leisure-time sitting, with cardiorespiratory and muscular fitness among working adults. Scand J Work Environ Health 2014;40(5):531-538. doi:10.5271/sjweh.3443

16. Martimo K-P, Varonen H, Husman K, Viikari-Juntura E. Factors associated with self-assessed work ability. Occup Med. 2007; 57(5): 380-2. doi:10.1093/occmed/ kqm028

17. Andersen LL, Fallentin N, Thorsen SV, Holtermann A. Physical workload and risk of long-term sickness absence in the general working population and among blue-collar workers: Prospective cohort study with register follow-up. Occup Environ Med. 2016; 73(4):246-253. doi:10.1136/oemed-2015-103314.

18. Lahelma $E$, Laaksonen $M$, Lallukka T, Martikainen $P$, Pietiläinen $O$, Saastamoinen $\mathrm{P}$, et al. Working conditions as risk factors for disability retirement: A longitudinal register linkage study. BMC Public Health. 2012;12(1): 309. doi:10.1186/14712458-12-309

19. Hannerz $H$, Tüchsen $F$, Spangenberg $S$, Albertsen $K$. Industrial differences in disability retirement rates in Denmark, 1996-2000. Int J Occup Med Environ Health. 2004;17(4):465-471.

20. Astudillo P, Ibarra C. La perspectiva de género, Desafíos para la Ergonomía en Chile: Una revisión Sistemática de Literatura. Ciencia \& trabajo. 2014; 16(49), 2837. doi:10.4067/S0718-24492014000100006

21. St-Vincent $M$, Vézina $N$, Bellemare $M$, Denis $D$, Ledoux E, Imbeau D. L'intervention en ergonomie. Montreal : Éditions Multimonde et IRSST; 2011. ISBN : 9782895441656 
22. Guérin F, Laville A, Daniellou F, Duraffourg J, Kerguelen A. Comprendre le travail pour le transformer: La pratique de l'ergonomie. III edition. Toulouse: Editions ANACT France; 2008. ISBN-10: 2913488498.

23. Marras WS. The working back: A systems view. I edition. Hoboken. John Wiley \& Sons, Inc.; 2008. ISBN: 978-0-470-13405-4

24. Chaffin DB, Andersson GBJ, Martin BJ. Occupational Biomechanics, IV edition. Hoboken. John Wiley \& Sons, Inc.; 2006. ISBN: 978-0-471-72343-1

25. Waters TR, Putz-Anderson V, Garg A, Fine LJ. Revised NIOSH equation for the design and evaluation of manual lifting tasks. Ergonomics. 1993;36(7):749-776. doi:10.1080/00140139308967940

26. McGill SM. The biomechanics of low back injury: Implications on current practice in industry and the clinic. J Biomech. 1997;30(5):465-75. doi:10.1016/S00219290(96)00172-8

27. Snook SH, Ciriello VM. The design of manual handling tasks: Revised tables of maximum acceptable weights and forces. Ergonomics. 1991;34(9):1197-213. doi:10.1080/00140139108964855

28. Ministerio del Trabajo y Previsión Social de Chile. Guía Técnica para la Evaluación y Control de Riesgos Asociados al Manejo o Manipulación Manual de Carga. Subsecretaría de Previsión Social de Chile. [online]. 2018. Disponible en URL: https://www.previsionsocial.gob.cl/sps/download/biblioteca/seguridad-y-salud-en-el-trabajo/guia-manejo-cargas/guia-tecnica-manejo-manual-de-carga.pdf

29. da Costa BR, Vieira ER. Risk factors for work-related musculoskeletal disorders: a systematic review of recent longitudinal studies. Am J Ind Med. 2009; 53(3): 285-323. doi:10.1002/ajim.20750

30. Holtermann A, Jørgensen MB, Gram B, Christensen JR, Faber A, Overgaard $\mathrm{K}$, et al. Worksite interventions for preventing physical deterioration among employees in job-groups with high physical work demands: Background, design and conceptual model of FINALE. BMC Public Health. 2010;10: 120. doi:10.1186/14712458-10-120.

31. Baltrusch SJ, van Dieën JH, Bruijn SM, Koopman AS, van Bennekom CAM, Houdijk $\mathrm{H}$. The effect of a passive trunk exoskeleton on metabolic costs during lifting and walking. Ergonomics. 2019; 62(7): 903-916. doi:10.1080/00140139.20 19.1602288

32. Ghezelbash F, Shirazi-Ad A, Plamondon A, Arjmand N, Parnianpour M. Obesity and obesity shape markedly influence spine biomechanics: a subject-specific risk assessment model. Ann Biomed Eng. 2017; 45, 2373-2382. doi:10.1007/s10439017-1868-7

33. Ghezelbash F, Shirazi-Adl A, Arjmand N, El-Ouaaid Z, Plamondon A, Meakin, JR. Effects of sex, age, body height and body weight on spinal loads: Sensitivity analyses in a subject-specific trunk musculoskeletal model. Journal of biomechanics. 2016; 49(14), 3492-3501. doi:10.1016/j.jbiomech.2016.09.026 